\title{
Correlações genéticas e fenotípicas entre características reprodutivas e produtivas de touros da raça Nelore
}

\author{
Juliano Cesar Dias ${ }^{(1)}$, Venício José de Andrade ${ }^{(2)}$, Jorge André Matias Martins ${ }^{(1)}$, Lucas Luz Emerick ${ }^{(1)}$ \\ e Vicente Ribeiro do Vale Filho ${ }^{(3)}$
}

\begin{abstract}
(1)Universidade Federal de Minas Gerais (UFMG), Escola de Veterinária, Avenida Antônio Carlos, no 6627, Caixa Postal 567, CEP $30123-970$ Belo Horizonte, MG. E-mail: julianocdias@yahoo.com.br, jammvet@gmail.com, lucas-emerick@bol.com.br (2)UFMG, Escola de Veterinária, Departamento de Zootecnia. E-mail: venicio@vet.ufmg.br (3)UFMG, Escola de Veterinária, Departamento de Clínica e Cirurgia. E-mail: clinica@vet.ufmg.br
\end{abstract}

\begin{abstract}
Resumo - O objetivo deste trabalho foi estimar correlações genéticas e fenotípicas de características reprodutivas e ponderais em 579 touros Nelore, em criação extensiva. As características reprodutivas estudadas foram: circunferência escrotal, consistência, volume e forma testiculares, comprimento dos testículos esquerdo e direito, largura dos testículos esquerdo e direito, motilidade e vigor espermáticos, defeitos espermáticos maiores, menores e totais e classificação andrológica por pontos. As características foram analisadas pelo método de máxima verossimilhança restrita, com algoritmos livres de derivadas, sob modelo animal, com inclusão da matriz de numeradores dos coeficientes de parentesco entre os animais e seus ascendentes. As correlações genéticas entre circunferência escrotal e as características peso corporal, volume testicular, motilidade espermática, vigor espermático, defeitos espermáticos menores, defeitos espermáticos totais e classificação andrológica por pontos foram, respectivamente, $0,72,0,99,0,72,0,60,-0,67,-0,12$ e 0,64 . As maiores correlações fenotípicas encontradas entre peso e circunferência escrotal, características físicas e morfológicas do sêmen, quando comparadas às correlações entre idade e as mesmas características, são indicativas de que o peso tem maior influência na condição reprodutiva. As correlações genéticas entre classificação andrológica por pontos e as características: peso, circunferência escrotal, volume testicular, defeitos espermáticos maiores e defeitos espermáticos totais foram, respectivamente, $0,19,0,64,0,71,-0,47 \mathrm{e}-0,58$.
\end{abstract}

Termos para indexação: Bos indicus, característica seminal, genética, reprodução.

\section{Genetic and phenotypic correlations among reproductive and productive traits of Nelore bulls}

\begin{abstract}
The aim of this work was to estimate genetic and phenotypic correlations for sexual reproductive and productive traits using a Multiple Trait Derivative-Free Restricted Maximum Likelihood (MTDFREML) methodology in 579 Nelore bulls, raised under pasture. The reproductive traits analyzed were scrotal circumference, testicular consistency, testicular volume, testicular shape, length and width of right and left testicles, physical (motility and vigor) and morphological (major and minor, and total sperm defects) semen traits, and breeding soundness evaluation. Genetic correlations between scrotal circumference and body weight, testicular volume, motility, vigor, minor sperm defects, total sperm defects and breeding soundness evaluation were, respectively, $0.72,0.99,0.72,0.60,-0.67,-0.12$ e 0.64 , showing favorable associations of scrotal circumference and the characteristics related. Phenotypic correlations of major magnitude found among ages and the above-related characteristics suggested to be the body weight the major factor affecting the reproductive performance of those animals. Genetic correlations among breeding soundness evaluation, body weight, scrotal circumference, testicular volume, major sperm defects and total sperm defects were, respectively, $0.19,0.64,0.71,-0.47 \mathrm{e}-0.58$.
\end{abstract}

Index terms: Bos indicus, seminal trait, genetic, reproduction.

\section{Introdução}

O valor comercial de um animal resulta do número de características desejáveis que influenciam o seu desempenho. A seleção de determinada característica é importante não somente pelos reflexos na sua expressão, como também na de outras que lhes são dependentes, em maior ou menor grau (Pereira, 2000).

A eficiência reprodutiva pode ser considerada uma das mais importantes características econômicas, e deve 
receber especial atenção dos criadores de gado de corte. A reprodução é um processo complexo e a seleção direta de características ligadas à reprodução é, muitas vezes, difícil de ser aplicada. Por isso, torna-se necessário identificar características que sejam facilmente mensuradas, que apresentem variabilidade e que sejam geneticamente correlacionadas aos eventos reprodutivos (Bergmann et al., 1997; Pereira, 2000). Dessa forma, a associação entre duas ou mais características, observadas em indivíduos de uma população, torna-se ferramenta nos processos de seleção.

No Brasil, existem poucos relatos a respeito das associações genéticas entre características ponderais, medidas testiculares e características seminais para touros de raças zebuínas (Bos taurus indicus). Entretanto, estudos (Bergmann et al., 1996, 1997; Quirino et al., 1999; Sarreiro et al., 2002) têm demonstrado resposta correlacionada em características seminais e ponderais quando se seleciona para circunferência escrotal.

O objetivo deste trabalho foi estimar correlações genéticas e fenotípicas de características reprodutivas e ponderais de touros da raça Nelore, em criação extensiva.

\section{Material e Métodos}

O trabalho foi desenvolvido na Fazenda Cristo, Município de Miranda, Estado do Mato Grosso do Sul, na região conhecida como Serra da Bodoquena, situada em área de transição entre Pantanal e Planalto Central.

Os dados referiram-se a características reprodutivas e produtivas de um total de 579 touros da raça Nelore, com idade de 19 a 39 meses, oriundos de um rebanho PO, de propriedade da Agropecuária Nove de Ouro, localizada no Município de Miranda, MS, avaliados entre os anos de 2000 e 2002.

As características reprodutivas avaliadas foram: biometrias testiculares (circunferência escrotal, CE; consistência testicular, CONS; volume testicular, VOLT; forma testicular, FORT; comprimento do testículo esquerdo, CTE; comprimento do testículo direito, CTD; largura do testículo esquerdo, LTE; largura do testículo direito, LTD), características físicas (motilidade, MOTIL e vigor) e morfológicas (defeitos espermáticos maiores, DM, menores, DMe e totais, DT) do sêmen, conforme descrito pelo Colégio Brasileiro de Reprodução Animal (1998); e classificação andrológica por pontos (CAP), segundo Vale Filho et al. (2003), para estabelecimento de correlações genéticas e fenotípicas entre essas características, e peso corporal (PESO) de animais Nelore, criados extensivamente.

Quanto às estimativas dos componentes de covariância, necessários às estimativas dos valores gênicos dos animais e das correlações genéticas e ambientes, foi utilizado o método de máxima verossimilhança restrita, com algoritmos livres de derivadas, sob modelo animal, com inclusão da matriz dos numeradores dos coeficientes de parentesco entre os animais e seus ascendentes. O modelo considerou o efeito das variáveis independentes: mês e ano de nascimento e idade do animal, todas usadas como efeitos fixos. Para tanto, utilizou-se o programa MTDFREML (Multiple Trait Derivative-Free Restricted Maximum Likelihood), descrito por Boldman et al. (1995).

Os critérios de convergência foram: valor de predição da variância do Simplex para localizar o mínimo de $-2 \log \mathrm{L} 10^{-6}$ (em que L é a função de máxima verossimilhança) em modelos com duas características; e não haver alteração no valor das estimativas do Simplex -2 Log de verossimilhança $10^{-9} \mathrm{em}$ duas repetições sucessivas. A cada ciclo o programa era reiniciado, e utilizaram-se como valores de co-variância iniciais os obtidos na análise anterior.

O modelo utilizado foi: $\mathrm{Y}=\mathrm{Xb}+\mathrm{Zu}+\mathrm{e}$, em que $\mathrm{Y}$ é o vetor de observações de características medidas nos indivíduos; X, Z são matrizes de incidências dos efeitos fixos e aleatórios, respectivamente; $b$ é o vetor de efeitos fixos; u é o vetor dos efeitos aleatórios de valores genético-aditivos diretos do animal; e é o vetor dos erros aleatórios associados a cada observação.

Estimativas dos coeficientes de correlação de Pearson (correlações simples) foram calculadas pelo Proc-Corr, utilizando-se o SAS (1996).

\section{Resultados e Discussão}

$\mathrm{Na}$ Tabela 1, são apresentadas as correlações genéticas entre as diferentes medidas testiculares e PESO. As magnitudes das correlações genéticas entre as características apresentaram-se favoráveis e altas, o que mostra base genética comum entre as características, conforme sugeriu Quirino et al. (1999).

A correlação genética favorável e de alta magnitude entre CE e PESO é indicação de que a CE é parâmetro adequado para identificação de touros com maiores potenciais de ganho de peso (Salvador et al., 2002). Resultado semelhante de correlação genética entre 
PESO e CE foi registrado por Quirino et al. (1999). Bergmann et al. (1996) encontraram correlações genéticas de menor magnitude entre essas características para animais de 12 e 18 meses de idade.

As correlações genéticas favoráveis e de alta magnitude entre VOLT e comprimento e largura dos testículos indicam a existência de base genética comum para estas características e de influência direta do comprimento e da largura sobre a massa testicular. Entretanto, correlações favoráveis e de alta magnitude entre VOLT, CTE, CTD, LTE, LTD e CE indicam que a CE é parâmetro adequado para ser utilizado nos programas de seleção para predição do tamanho dos testículos em animais da raça Nelore. Viu et al. (2006) encontraram valor de correlação entre CE e volume testicular de 0,81 , e sugeriram que a seleção para precocidade sexual por meio da CE levaria à resposta correlacionada no volume testicular. Unanian et al.
(2000) observaram que a CE é mais influenciada pela largura do que pelo comprimento dos testículos, o que poderia comprometer essa associação em animais de testículos longos.

Correlações genéticas altas e favoráveis entre CE, VOLT, CTE, CTD, LTE, LTD e peso corporal indicam compatibilidade de seleção para crescimento corporal e fertilidade nos programas de seleção de reprodutores da raça Nelore. Touros com maiores CE apresentam maiores massas testiculares e maiores pesos corporais, conforme Quirino et al. (1999).

$\mathrm{Na}$ Tabela 2, são apresentadas as correlações fenotípicas entre as diferentes características biométricas testiculares e PESO. Observam-se correlações altas e favoráveis entre idade e as demais características, nesse grupamento de animais estudado, o que indica aumento dessas características com o aumento da idade.

Tabela 1. Correlações genéticas entre diversas características biométricas testiculares em touros Nelore.

\begin{tabular}{|c|c|c|c|c|c|c|c|c|}
\hline Característica $^{(1)}$ & $\begin{array}{c}\mathrm{CE} \\
(\mathrm{cm})\end{array}$ & $\begin{array}{l}\text { VOLT } \\
\left(\mathrm{cm}^{3}\right)\end{array}$ & $\begin{array}{c}\text { CONS } \\
(1-5)\end{array}$ & $\begin{array}{r}\text { FORT } \\
(1-5)\end{array}$ & $\begin{array}{l}\mathrm{CTE} \\
(\mathrm{cm})\end{array}$ & $\begin{array}{l}\text { CTD } \\
(\mathrm{cm})\end{array}$ & $\begin{array}{l}\mathrm{LTE} \\
(\mathrm{cm})\end{array}$ & $\begin{array}{l}\text { LTD } \\
(\mathrm{cm})\end{array}$ \\
\hline PESO & 0,72 & 0,75 & 0,28 & 0,80 & 0,44 & 0,32 & 0,84 & 0,84 \\
\hline $\mathrm{CE}$ & - & 0,99 & 0,17 & 0,39 & 0,91 & 0,81 & 0,96 & 0,96 \\
\hline OLT & - & - & 0,43 & 0,30 & 0,86 & 0,83 & 0,99 & 0,99 \\
\hline ONS & - & - & - & 1,00 & $-0,24$ & $-0,36$ & 0,43 & 0,60 \\
\hline ORT & - & - & - & - & $-0,31$ & $-0,40$ & 0,46 & 0,56 \\
\hline CTE & - & - & - & - & - & 1,00 & 0,75 & 0,79 \\
\hline LTE & - & - & - & - & - & - & - & 1,00 \\
\hline
\end{tabular}

(1)PESO, peso corporal; CE, circunferência escrotal; VOLT, volume testicular; CONS, consistência testicular; FORT, forma testicular; CTE, comprimento testículo esquerdo; CTD, comprimento testículo direito; LTE, largura testículo esquerdo; LTD, largura testículo direito.

Tabela 2. Correlações fenotípicas entre diversas características biométricas testiculares em touros Nelore.

\begin{tabular}{|c|c|c|c|c|c|c|c|}
\hline Característica $^{(1)}$ & $\begin{array}{c}\text { Idade } \\
\text { (meses) }\end{array}$ & $\begin{array}{l}\text { PESO } \\
(\mathrm{kg})\end{array}$ & $\begin{array}{l}\mathrm{CE} \\
(\mathrm{cm})\end{array}$ & $\begin{array}{l}\text { VOLT } \\
\left(\mathrm{cm}^{3}\right)\end{array}$ & $\begin{array}{l}\text { CTE } \\
(\mathrm{cm})\end{array}$ & $\begin{array}{l}\text { CTD } \\
(\mathrm{cm})\end{array}$ & $\begin{array}{l}\text { LTE } \\
(\mathrm{cm})\end{array}$ \\
\hline \multirow[t]{2}{*}{ PESO } & 0,77 & - & - & - & - & - & - \\
\hline & $(0,0001)^{*}$ & - & - & - & - & - & - \\
\hline \multirow[t]{2}{*}{$\mathrm{CE}$} & 0,67 & 0,74 & - & - & - & - & - \\
\hline & $(0,0001)$ & $(0,0001)$ & - & - & - & - & - \\
\hline \multirow[t]{2}{*}{ VOLT } & 0,71 & 0,77 & 0,93 & - & - & - & - \\
\hline & $(0,0001)$ & $(0,001)$ & $(0,0001)$ & - & - & - & - \\
\hline \multirow[t]{2}{*}{ CTE } & 0,52 & 0,59 & 0,80 & 0,83 & - & - & - \\
\hline & $(0,0001)$ & $(0,0001)$ & $(0,0001)$ & $(0,0001)$ & & - & - \\
\hline \multirow[t]{2}{*}{ CTD } & 0,50 & 0,58 & 0,79 & 0,82 & 0,99 & - & - \\
\hline & $(0,0001)$ & $(0,0001)$ & $(0,0001)$ & $(0,0001)$ & $(0,0001)$ & - & - \\
\hline \multirow[t]{2}{*}{ LTE } & 0,70 & 0,75 & 0,92 & 0,96 & 0,73 & 0,71 & - \\
\hline & $(0,0001)$ & $(0,0001)$ & $(0,0001)$ & $(0,0001)$ & $(0,0001)$ & $(0,0001)$ & - \\
\hline \multirow[t]{2}{*}{ LTD } & 0,69 & 0,75 & 0,92 & 0,96 & 0,72 & 0,71 & 0,97 \\
\hline & $(0,0001)$ & $(0,0001)$ & $(0,0001)$ & $(0,0001)$ & $(0,0001)$ & $(0,0001)$ & $(0,0001)$ \\
\hline
\end{tabular}

(1)PESO, peso corporal; CE, circunferência escrotal; VOLT, volume testicular; CTE, comprimento testículo esquerdo; CTD, comprimento testículo direito; LTE, largura testículo esquerdo; LTD, largura testículo direito. *Nível de significância. 
Houve pequeno aumento dos valores das correlações fenotípicas entre PESO e CE, VOLT, CTE e CTD comparados aos valores de correlações genéticas, porém com valores menores para correlações entre PESO e LTE e LTD. Correlações genéticas e fenotípicas entre PESO e CE, VOLT, LTE, LTD, CTE, CTD, CONS apresentaram-se favoráveis e de moderada a alta magnitude, indicando influência da condição corporal nessas características reprodutivas. Os valores foram semelhantes aos encontrados na literatura (Bergmann et al., 1997; Quirino et al., 1999; Salvador et al., 2002).

As correlações fenotípicas entre CE e VOLT, CTD, LTE e LTD são semelhantes aos valores encontrados para correlações genéticas, porém com valores inferiores e similares aos relatados por Freneau et al. (2006).

A estimativa de correlação fenotípica entre CE e idade (Tabela 2) mostrou-se favorável e de alta magnitude, o que indica que a seleção por meio da CE poderia identificar animais mais precoces.

Os valores das correlações fenotípicas encontrados entre VOLT e comprimento e largura dos testículos (Tabela 2) indicam a influência dessas características sobre o volume testicular, e ainda a correlação de maior magnitude entre a largura dos testículos e VOLT denota sua maior influência na determinação dessa característica.

Entretanto, as correlações de alta magnitude entre CE e as demais características biométricas testiculares são indicação de que a CE é medida recomendável para expressar o volume testicular em touros da raça Nelore.

As correlações fenotípicas de maiores magnitudes encontradas entre PESO e características de biometria testicular, comparadas às correlações entre idade e características de biometria testicular, são indicação de que o PESO tem maior influência no desenvolvimento testicular desses animais.

$\mathrm{Na}$ Tabela 3, são apresentadas as correlações genéticas entre PESO, características de biometria testicular, características físicas e morfológicas do sêmen e CAP. As estimativas de correlações genéticas entre peso corporal e características físicas do sêmen apresentaram-se baixa e moderada, entre PESO e vigor $(0,02)$ e entre PESO e motilidade espermática $(0,22)$, e estes resultados são diferentes dos encontrados por Freneau et al. (2006) para correlações fenotípicas, que relataram de 0,63 entre PESO e vigor e de 0,70 entre PESO e motilidade.

Quando consideradas as correlações genéticas entre peso corporal e características morfológicas do sêmen (DM, DMe e DT) (Tabela 3), registraram-se correlações favoráveis porém de baixa magnitude o que destaca a importância do peso corporal nas características morfológicas seminais.

As correlações genéticas entre CE e características físicas do sêmen (Tabela 3) apresentaram-se favoráveis e de alta magnitude, 0,72 entre CE e motilidade e 0,60 entre CE e vigor, o que mostra a importância da CE nas características físicas estudadas. Existem resultados diversos de correlações genéticas entre CE e motilidade, variando de 0,13 a 1,00 (Gipson et al., 1987; Bergmann et al., 1997; Quirino et al., 1999; Sarreiro et al., 2002); já os resultados de correlações genéticas entre CE e vigor variaram de 0,69 a 0,99 (Bergmann et al., 1997; Quirino et al., 1999; Sarreiro et al., 2002). Neste trabalho, a correlação entre as características físicas do sêmen (motilidade e vigor) também apresentou valor alto $(0,65)$, o que denota base genética comum para as características.

Tabela 3. Correlações genéticas entre peso, características biométricas testiculares, características físicas e morfológicas do sêmen e CAP em touros Nelore.

\begin{tabular}{lcccccccrr}
\hline Característica $^{(1)}$ & $\begin{array}{c}\text { CE } \\
(\mathrm{cm})\end{array}$ & $\begin{array}{c}\text { CONS } \\
(1-5)\end{array}$ & $\begin{array}{c}\text { VOLT } \\
\left(\mathrm{cm}^{3}\right)\end{array}$ & $\begin{array}{c}\text { MOTIL } \\
(\%)\end{array}$ & $\begin{array}{r}\text { Vigor } \\
(1-5)\end{array}$ & $\begin{array}{r}\text { DM } \\
(\%)\end{array}$ & $\begin{array}{r}\text { DMe } \\
(\%)\end{array}$ & $\begin{array}{r}\text { DT } \\
(\%)\end{array}$ & $\begin{array}{r}\text { CAP } \\
(0-100)\end{array}$ \\
\hline PESO & 0,72 & 0,28 & 0,75 & 0,22 & 0,02 & $-0,10$ & $-0,19$ & $-0,10$ & 0,19 \\
CE & - & 0,17 & 0,99 & 0,72 & 0,60 & 0,13 & $-0,67$ & $-0,12$ & 0,64 \\
CONS & - & - & 0,43 & $-0,14$ & $-0,79$ & 0,15 & 0,32 & 0,21 & $-0,17$ \\
VOLT & - & - & - & 0,68 & 0,54 & 0,12 & $-0,59$ & $-0,08$ & 0,71 \\
MOTIL & - & - & - & - & 0,65 & 0,00 & $-1,00$ & $-0,71$ & 1,00 \\
Vigor & - & - & - & - & - & $-0,78$ & $-1,00$ & $-1,00$ & 1,00 \\
DM & - & - & - & - & - & - & $-0,39$ & 0,95 & $-0,47$ \\
DMe & - & - & - & - & - & - & - & $-0,08$ & $-0,07$ \\
DT & - & - & - & - & - & - & - & - & $-0,58$ \\
\hline
\end{tabular}

(1)PESO, peso corporal; CE, circunferência escrotal; CONS, consistência testicular; VOLT, volume testicular; MOTIL, motilidade espermática; DM, defeito espermático maior; Dme, defeito espermático menor; DT, defeito espermático total; CAP, classificação andrológica por pontos. 
As correlações genéticas entre $\mathrm{CE}$ e defeitos espermáticos menores (DMe) e totais (DT) apresentaram valores negativos e, respectivamente, de alta e baixa magnitude, o que indica associação favorável entre o desenvolvimento testicular e as características morfológicas desejáveis do sêmen. Todavia, a correlação genética entre CE e DM apresentou valor oposto aos encontrados na literatura (Bergmann et al., 1997; Quirino et al., 1999).

Os resultados de correlações genéticas encontrados entre CE e características físicas (motilidade e vigor) e morfológicas (DMe e DT) do sêmen são indicação da base genética comum entre elas, e que a seleção de reprodutores baseada na $\mathrm{CE}$ leva à seleção indireta favorável das características seminais; resultado semelhante ao encontrado por Sarreiro et al. (2002).

Correlações genéticas observadas entre VOLT e as características estudadas (Tabela 3 ) foram, na sua maioria, inferiores às encontradas para $\mathrm{CE}$ e estas mesmas características, o que mostra que a CE é parâmetro adequado de seleção para touros da raça Nelore.

A estimativa de correlação genética entre CAP e PESO apresentou valor de baixa magnitude. Já as correlações genéticas entre CE, VOLT e CAP mostram a importância do desenvolvimento dos testículos neste índice de classificação de reprodutores. A CAP apresentou correlações genéticas negativas e de alta magnitude, com DM e DT $(-0,47$ e $-0,58$, respectivamente), o que é indicação da importância da morfologia espermática nesse índice de classificação de reprodutores, conforme sugeriram Vale Filho et al. (1999). Portanto, é importante a inclusão da análise de sêmen com a CE na avaliação de touros jovens.

$\mathrm{Na}$ Tabela 4, são apresentadas as correlações fenotípicas entre PESO, características de biometria testicular, características físicas e morfológicas do sêmen e CAP, em touros Nelore. As correlações fenotípicas foram, na sua maioria, maiores que as correlações genéticas, resultado diferente do encontrado por Quirino et al. (1999).

As correlações fenotípicas entre CE e características físicas e morfológicas do sêmen apresentaram-se com maiores magnitudes que as encontradas por Martinez et al. (2000) para reprodutores da raça Gir, selecionados para leite, em centrais de coleta de sêmen.

As correlações de maiores magnitudes encontradas entre PESO e características físicas e morfológicas do sêmen, quando comparadas às correlações entre idade e as mesmas características, são indicação de que o

Tabela 4. Correlações fenotípicas entre peso, características biométricas testiculares, características físicas e morfológicas do sêmen e CAP em touros Nelore.

\begin{tabular}{|c|c|c|c|c|c|c|c|c|c|c|}
\hline Característica $^{(1)}$ & $\begin{array}{c}\text { Idade } \\
\text { (meses) }\end{array}$ & $\begin{array}{l}\text { PESO } \\
(\mathrm{kg})\end{array}$ & $\begin{array}{l}\mathrm{CE} \\
(\mathrm{cm})\end{array}$ & $\begin{array}{c}\text { CONS } \\
(1-5)\end{array}$ & $\begin{array}{l}\text { VOLT } \\
\left(\mathrm{cm}^{3}\right)\end{array}$ & $\begin{array}{c}\text { MOTIL } \\
(\%)\end{array}$ & $\begin{array}{l}\text { Vigor } \\
(1-5)\end{array}$ & $\begin{array}{l}\text { DM } \\
(\%)\end{array}$ & $\begin{array}{c}\mathrm{DMe} \\
(\%)\end{array}$ & $\begin{array}{l}\text { DT } \\
(\%)\end{array}$ \\
\hline \multirow[t]{2}{*}{ PESO } & 0,77 & - & - & - & - & - & - & - & - & - \\
\hline & $(0,0001)^{*}$ & - & - & - & - & - & - & - & - & - \\
\hline \multirow[t]{2}{*}{$\mathrm{CE}$} & 0,67 & 0,74 & - & - & - & - & - & - & - & - \\
\hline & $(0,0001)$ & $(0,0001)$ & - & - & - & - & - & - & - & - \\
\hline \multirow[t]{2}{*}{ CONS } & 0,10 & 0,23 & 0,23 & - & - & - & - & - & - & - \\
\hline & $(0,0009)$ & $(0,0001)$ & $(0,0001)$ & - & - & - & - & - & - & - \\
\hline \multirow[t]{2}{*}{ VOLT } & 0,71 & 0,77 & 0,93 & 0,18 & - & - & - & - & - & - \\
\hline & $(0,0001)$ & $(0,0001)$ & $(0,0001)$ & $(0,0001)$ & - & - & - & - & - & - \\
\hline \multirow[t]{2}{*}{ MOTIL } & 0,06 & 0,04 & 0,16 & $-0,02$ & 0,13 & - & - & - & - & - \\
\hline & $(0,0807)$ & $(0,2906)$ & $(0,0001)$ & $(0,4774)$ & $(0,0001)$ & - & - & - & - & - \\
\hline \multirow[t]{2}{*}{ Vigor } & 0,01 & 0,04 & 0,14 & $-0,02$ & 0,12 & 0,81 & - & - & - & - \\
\hline & $(0,6348)$ & $(0,2082)$ & $(0,0001)$ & $(0,4661)$ & $(0,0007)$ & $(0,0001)$ & & - & - & - \\
\hline \multirow[t]{2}{*}{$\mathrm{DM}$} & $-0,03$ & $-0,15$ & $-0,13$ & $-0,10$ & $-0,09$ & $-0,28$ & $-0,23$ & - & - & - \\
\hline & $(0,2776)$ & $(0,0001)$ & $(0,0002)$ & $(0,0048)$ & $(0,013)$ & $(0,0001)$ & $(0,0001)$ & - & - & - \\
\hline \multirow[t]{2}{*}{$\mathrm{DMe}$} & $-0,05$ & $-0,002$ & $-0,07$ & $-0,03$ & $-0,09$ & $-0,18$ & $-0,12$ & 0,01 & - & - \\
\hline & $(0,115)$ & $(0,9553)$ & $(0,0329)$ & $(0,3731)$ & $(0,0064)$ & $(0,0001)$ & $(0,0004)$ & $(0,6882)$ & - & - \\
\hline \multirow[t]{2}{*}{ DT } & $-0,05$ & $-0,13$ & $-0,15$ & $-0,10$ & $-0,12$ & $-0,33$ & $-0,26$ & 0,91 & 0,41 & - \\
\hline & $(0,1051)$ & 0,0006 & $(0,0001)$ & $(0,0034)$ & $(0,0008)$ & $(0,0001)$ & $(0,0001)$ & $(0,0001)$ & $(0,0001)$ & - \\
\hline CAP & 0,06 & 0,16 & 0,36 & 0,05 & 0,26 & 0,32 & 0,30 & $-0,69$ & $-0,19$ & $-0,65$ \\
\hline
\end{tabular}

(1)PESO, peso corporal; CE, circunferência escrotal; CONS, consistência testicular; VOLT, volume testicular; MOTIL, motilidade espermática; DM, defeito espermático maior; Dme, defeito espermático menor; DT, defeito espermático total; CAP, classificação andrológica por pontos. *Nível de significância. 
PESO exerce maior influência na condição reprodutiva desses animais.

As correlações fenotípicas entre DM, DT e CAP foram negativas e de alta magnitude, o que sugere a influência dessas características na determinação da pontuação desses animais, com valares maiores que os encontrados por Salvador et al. (2002).

Fonseca (1989), Bergmann et al. (1996), Vale Filho et al. (1997), Quirino et al. (1999), Gressler et al. (2000) e Sarreiro et al. (2002) sugerem que a CE é uma característica reprodutiva importante, por ser indicativa de maior produção espermática diária, precocidade reprodutiva, por apresentar alta correlação com peso corporal, por ser correlacionada com características reprodutivas de fêmeas bovinas e ainda por ser medianamente correlacionada com características indicativas de qualidade espermática. Alguns desses resultados foram observados neste trabalho.

\section{Conclusões}

1. As correlações genéticas favoráveis entre circunferência escrotal e características físicas e morfológicas do sêmen, além da correlação genética de alta magnitude entre circunferência escrotal e peso, são indicação de que a circunferência escrotal é parâmetro adequado e pode ser usada como critério de seleção em programas de melhoramento visando maiores crescimento corporal e fertilidade.

2. As correlações genéticas favoráveis e de moderada a alta magnitude entre o índice de classificação andrológica por pontos (CAP) e peso, circunferência escrotal, defeitos espermáticos maiores e totais são indicação de que esse índice pode se utilizado como critério de seleção de touros Nelore.

\section{Referências}

BERGMANN, J.A.G.; ZAMBORLINI, L.C.; PROCÓPIO, C.S.O.; ANDRADE, V.J.; VALE FILHO, V.R. Estimativas de parâmetros genéticos do perímetro escrotal e do peso corporal em animais da raça Nelore. Arquivo Brasileiro de Medicina Veterinária e Zootecnia, v.48, p.69-78, 1996.

BERGMANN, J.A.G.; QUIRINO, C.R.; VALE FILHO, V.R.; ANDRADE, V.J.; FONSECA, C.G. Herdabilidades e correlações genéticas entre medições testiculares e características espermáticas em touros Nelore. Archivos Latinoamericanos de Producción Animal, v.5, p.473-475, 1997. Suplemento 1.

BOLDMAN, K.G.; KRIESE, L.A.; VAN VLECK, L.D.; KACHMAN, S.D. A manual for use for MTDFREML: a set of programs to obtain estimates of variance and covariance [DRAFT]. Lincoln: Departament of Agriculture/Agricultural Research Service, 1995. 120p.

COLÉGIO BRASILEIRO DE REPRODUÇÃO ANIMAL. Manual para exame andrológico e avaliação de sêmen animal. 2.ed. Belo Horizonte: CBRA, 1998.

FONSECA, V.O. Puberdade, adolescência e maturidade sexual: aspectos histopatológicos e comportamentais. In: CONGRESSO BRASILEIRO DE REPRODUÇÃO ANIMAL, 8., 1989, Belo Horizonte. Anais. Belo Horizonte: Colégio Brasileiro de Reprodução Animal, 1989. p.77-93.

FRENEAU, G.E.; VALE FILHO, V.R.; MARQUES JUNIOR, A.P.; MARIA, W.S. Puberdade em touros Nelore criados em pasto no Brasil: características corporais, testiculares e seminais e de índice de capacidade andrológica por pontos. Arquivo Brasileiro de Medicina Veterinária e Zootecnia, v.58, p.1107-1115, 2006.

GIPSON, T.A.; VOGT, D.W.; ELLERSIECK, M.R.; MASSEY, J.W. Genetic and phenotypic next term parameter estimates for scrotal circumference and semen traits in young beef bulls. Theriogenology, v.28, p.547-555, 1987.

GRESSLER, S.L.; BERGMANN, J.A.G.; PENNA, V.M.; PEREIRA, C.S.; PEREIRA, J.C.C.; GRESSLER, M.G.M. Estudo das associações genéticas entre perímetro escrotal e características reprodutivas de fêmeas Nelore. Revista Brasileira de Zootecnia, v.29, p.427-437, 2000.

MARTINEZ, M.L.; VERNEQUE, R.S.; TEODORO, R.L.; PAULA, L.R.O.; CRUZ, M.; CAMPOS, J.P.; RODRIGUES, L.H.; OLIVEIRA, J.; VIEIRA, F.; BRUSCHI, J.H.; DURÃES, M.C. Correlações entre características da qualidade do sêmen e a circunferência escrotal de reprodutores da raça Gir. Revista Brasileira de Zootecnia, v.29, p.700-706, 2000.

PEREIRA, J.C.C. Contribuição genética do zebu na pecuária bovina do Brasil. Informe Agropecuário, v.21, p.30-38, 2000.

QUIRINO, C.R.; BERGMANN, J.A.G.; VALE FILHO, V.R.; ANDRADE, V.J.; REIS, S.R.; MENDONÇA, R.; FONSECA, C.G. Genetic parameters of scrotal circunference, seminal characteristics and libido in Brazilian zebu bulls. Revista Brasileira de Reprodução Animal, v.23, p.252-253, 1999.

SALVADOR, D.F.; DIAS, J.C.; VALE FILHO, V.R.; ANDRADE, V.J.; SILVA, A.S., NOGUEIRA, E. Perfil andrológico de touros da raça Nelore com três e quatro anos de idade, criados extensivamente em condições do Estado do Mato Grosso do Sul. Revista Brasileira de Reprodução Animal, v.26, p.64-67, 2002.

SARREIRO, L.C.; BERGMANN, J.A.G.; QUIRINO, C.R.; PINEDA, N.R.; FERREIRA, V.C.P.; SILVA, M.A. Herdabilidade e correlação genética entre perímetro escrotal, libido e características seminais de touros Nelore. Arquivo Brasileiro de Medecina Veterinária e Zootecnia, v.54, p.602-608, 2002.

SAS INSTITUTE. User's Guide. Cary: SAS Institute, 1996.

UNANIAN, M.M.; SILVA, A.E.D.F.; MCMANUS, C.; CARDOSO, E.P. Características biométricas testiculares para 
avaliação de touros zebuínos da raça Nelore. Revista Brasileira de Zootecnia, v.29, p.136-144, 2000.

VALE FILHO, V.R.; ANDRADE, V.J.; SALVADOR, D.F.; DIAS, J.C.; ABREU, J.J.; GRAÇA, D.S.; BERGMANN, J.A.G. Prevalência de tourinhos da raça Tabapuã precoces e super-precoces (um e dois anos de idade), com base no perfil andrológico, submetidos a dois manejos nutricionais, na região de Nanuque, MG. Revista Brasileira de Reprodução Animal, v.27, p.178-180, 2003.

VALE FILHO, V.R.; BERGMANN, J.A.G.; ANDRADE, V.J.; QUIRINO, C.R.; REIS, S.R.; MENDONÇA, R.M.A. Caracterização andrológica de touros Nelore, selecionados para primeira estação de monta. Revista Brasileira de Reprodução Animal, v.21, p.42-45, 1997.
VALE FILHO, V.R.; QUIRINO,C.R.; ANDRADE, V.J.; BERGMANN, J.A.G.; REIS, S.R.; MENDONÇA, R.M.A.; FONSECA, C.G. Parâmetros genéticos da classificação andrológica por pontos (CAP), em touros da raça Nelore. Revista Brasileira de Reprodução Animal, v.23, p.253-255, 1999.

VIU, M.A.O.; MAGNABOSCO, C.U.; FERRAZ, H.T.; GAMBARINI, M.L.; OLIVEIRA FILHO, B.D.; LOPES, D.T.; VIU, A.M.F. Desenvolvimento ponderal, biometria testicular e qualidade seminal de touros Nelore (Bos taurus indicus) criados extensivamente na Região Centro-Oeste do Brasil. Archives of Veterinary Science, v.11, p.53-57, 2006.

Recebido em 30 de agosto de 2007 e aprovado em 28 de dezembro de 2007 